\title{
Reuse of Waste Energy from Power Plants in Greenhouses through MAS-Based Architecture
}

\author{
Alfonso González-Briones $\mathbb{D}^{\mathrm{D}},{ }^{1}$ Pablo Chamoso ${ }^{\mathbb{D}},{ }^{1}$ Sara Rodríguez, \\ Hyun Yoe $\mathbb{1}^{2},{ }^{2}$ and Juan M. Corchado ${ }^{1,3,4}$ \\ ${ }^{1}$ BISITE Digital Innovation Hub, University of Salamanca, Calle Espejo 2, 37007 Salamanca, Spain \\ ${ }^{2}$ School of Information and Communication Engineering, Sunchon National University, Suncheon, Republic of Korea \\ ${ }^{3}$ Department of Electronics, Information and Communication, Faculty of Engineering, Osaka Institute of Technology, \\ 535-8585 Osaka, Japan \\ ${ }^{4}$ Pusat Komputeran dan Informatik, Universiti Malaysia Kelantan, Karung Berkunci 36, Pengkaan Chepa, \\ 16100 Kota Bharu, Kelantan, Malaysia
}

Correspondence should be addressed to Alfonso González-Briones; alfonsogb@usal.es

Received 16 April 2018; Accepted 16 July 2018; Published 14 August 2018

Academic Editor: Alfonso Ramallo

Copyright (C) 2018 Alfonso González-Briones et al. This is an open access article distributed under the Creative Commons Attribution License, which permits unrestricted use, distribution, and reproduction in any medium, provided the original work is properly cited.

\begin{abstract}
Today, most energy-intensive processes have a high degree of optimization. However, in some of these processes large amounts of energy are inevitably released due to the way in which this energy is used. One of the most obvious examples is produced in the power plants during the process of obtaining electrical energy through the transformation of some kind of energy (chemical, kinetic, thermal, lighting, and nuclear or solar energy, among others). This released energy can be used in other processes that may need it so that no additional energy is needed. One of the possible uses of this energy is its use in greenhouses. Greenhouses need large amounts of energy to recreate the climatic conditions that crops need, which are not those of the weather station. To take advantage of the energy released from the power stations in greenhouses, a system based on agents has been developed that manages energy and allows it to be reused. This paper explains how the system allows us to reuse energy by a power plant and how the agents that integrate the system by means of communication with sensors and actuators and the use of data analysis algorithms allow us to use this energy in greenhouses, providing a reduction of the energy they need without the system. The system has been tested in several greenhouses with a pepper crop.
\end{abstract}

\section{Introduction}

Today, it is necessary to develop new ways of optimizing energy resources [1]. However, there are processes that by their characteristics release energy in the development of its activity, regardless of its optimization. This energy is commonly referred to as residual energy and one of the clearest examples is produced in power plants. In the power plants, it is produced during the process of obtaining electrical energy through the transformation of chemical, kinetic, thermal, light, nuclear, or solar energy, among others, in a large number of ways [2].

Many authors have focused on developing waste energy recovery systems and using it in other processes. However, there are no public studies on the reuse of energy from power plants. Bonilla et al. [3] discussed the potential of different WHR technologies for using the waste heat from the industry located in the Basque country in Spain. Al-Rabghi et al.[4] reviewed industrial WHRS for power production and process heating purposes. However, the study that most closely approximates our energy reuse proposal is the approach developed by $\mathrm{Hu}$ et al. in which they present a system that recovered the industrial waste heat to meet the demand for district heating [5]. High-efficiency centrifugal heat pumps are applied for district heating and heat recovery. A permanent-magnetic synchronous frequency-convertible (PSF) centrifugal heat pump is developed which has a much higher COP in comparison with that of conventional heat pumps. 
Greenhouses have high energy requirements because of the need to provide suitable climatic conditions for the development of off-season crops. There will be a high expenditure on electricity and/or gas if it is necessary to use the heating to have warm temperatures with respect to the outside or there will be an energy expenditure due to the use of cooling and ventilation systems when the temperature is below the outside temperature of the greenhouse. Due to these broad energy needs for optimal crop growth, greenhouses are an ideal focus for applying waste energy recovery systems in combination with power plant transformation processes. One of the locations where there is a great evolution in the greenhouse sector is in southwest Korea (South Jeolla). This region has orographical and climatic characteristics ideal for the cultivation of rice, wheat, barley, beans, or potatoes. However, this location requires the importation of many other agricultural products, which is why there is a great investment in the development of energy efficient greenhouses to solve these deficiencies. Therefore, the development of a system that allows further reducing energy consumption thanks to the use of unused energy will create optimal conditions, avoid negative effects, and maintain product quality by reducing the energy bill.

In order to recover and recycle the energy wasted by the plants, it is necessary to develop an approach that uses mechanisms to recover heat from hot flows. In this respect, there is work such as that carried out by Wang et al. in which waste heat is recovered in the form of hot water [6]. The hot water is obtained from the heat wasted in the different processes of the power plants, distributing it efficiently to the greenhouses, according to multiple parameters, so that its efficiency improves when establishing the desired temperature.

Due to the high costs involved in the deployment of an infrastructure of these characteristics, it is necessary to carry out a prior evaluation of the benefits provided by the system proposed in this work. To this end, the information has been transferred in terms of power dissipated by a power plant and simulated on a real infrastructure, which has not yet been connected to said power plant. The infrastructure used divides this energy among several greenhouses belonging to Sunchon National University.

However, to distribute the energy dissipated by the plant efficiently between greenhouses, it is necessary to deploy a system that collects information from both the plant and the inventories, to be the largest amount of energy dissipated by the plant but just so that it does not increase the temperature so that instead of benefit it produces a damage. In this sense, the environmental technology [7] is really useful in this task, since it allows adapting technology to people, making it possible to respond to their requirements intelligently and facilitating daily tasks. To perform autonomous actions, the problem can be faced by the multiagent systems (MAS); this approach is based on the agent technology [8], which is really relevant when trying to achieve common objectives in a distributed way within the framework of environmental intelligence. By integrating intelligent and dynamic mechanisms to learn from past experiences, the proposed architecture is able to provide users with better tools to save energy more efficiently. In particular, this paper presents a MAS-based platform whose purpose is the intelligent management of the energy released by the centers in a way that allows improving the use and distribution of hot water from waste heat recovery systems towards a whole of greenhouses. The multiagent system serves as an ideal framework for the development of agents that use AI algorithms such as information fusion (IF), case-based reasoning (CBR) system, and the use of traditional techniques of communication agents, cooperation, and negotiation.

The proposed system is designed to cover all aspects that are involved in a problem of this nature. On the one hand, the multiagent system provides a set of agents that are in charge of infrastructure management, information communication, sensor data capture, and agents that implement different AI algorithms as a neural network to model temperature variation. The system also includes a graphical interface that allows greenhouse farmers to know the energy that is reused in each greenhouse in real time.

The rest of the article is structured as follows. Section 2 presents an overview related to the reuse of energy. Section 3 provides a global description of the system, necessary infrastructure, and integration as well as the MAS. Section 4 presents the case study, including the results obtained. Finally, the conclusions obtained from the use of the system are presented.

\section{Overview}

Energy supply and waste management are important challenges that researches have faced for decades. During the last years, big progress has occurred but these issues are still relevant nowadays and new advantages can be gained in multiple areas [9]. This research work leverages new energy recovery technologies to distribute the energy obtained from waste energy of power plants, generally in the form of hot gas, to raise the temperature in greenhouses that need it. The aim is to optimize the use of energy and, thus, decrease the costs involved in maintaining a greenhouse.

Regarding the greenhouse farming, it is an expanding field because needs for agricultural products continue to increase together with population growth [10]. Using waste energy recovery systems for greenhouses is really important because it achieves a reduction of gas emissions through two separate mechanisms: (i) by generating electrical power or steam, using a waste energy recovery system avoids $\mathrm{CO}_{2}$ emissions from fossil fuel-based electrical generation; (ii) the combustion process the recovery system uses eliminates all potential methane emissions from landfill, thereby avoiding any potential release of methane in future. So an important reduction of gas emissions can be achieved. So far, greenhouse climate control has been improved only by using feedback-feed-forward control systems in combination with control algorithms and online adaptation of model parameter [11]; however the issue of energy efficiency in greenhouses is only a recent concern. Recent researches have focused on measuring the efficiency of energy use in greenhouses [12] and on the evaluation of using various renewable energy 
sources for heating a greenhouse [10], but there are no projects in the state of the art that would use the energy wasted produced by other industries.

Other recent researches focus on obtaining hot water from flue gases [6]; the study develops a new waste heat and water recovery technology.

2.1. Agents and Information Fusion. Agent technology has acquired a great importance in the field of distributed AI during last years. There are plenty of complexities in software agents [13]. MAS are groups of agents that allow solving problems in a distributed manner by making good use of the social and individual behaviors of the agents [14], which provides a natural method of characterizing intelligent systems. Therefore, when using a MAS, the concept of a single agent is expanded to include both the infrastructure for inter-action and the communication among them. MAS should include the following characteristics, as defined in [15]: (i) they have typically an open decentralized design; (ii) autonomous, heterogeneous, and distributed agents are included and each one has its own "personality" (cooperative, honest, selfish, etc.). The design includes the infrastructure specifically focused on the communication and interaction protocols, and the open characteristic should allow the participation of heterogeneous agents with different architectures or languages [16].

Another characteristic of some MAS is their organizational capability. It allows coordinating and exchanging services and information, for example, to negotiate and come to an agreement, so more complex social actions can be carried out. For this reason, research focused on the design of MAS from organizational perspectives seems to be gaining most ground. A VO is an open system that has been formed by the grouping and collaboration of heterogeneous entities (agents in this case). Agents organizations depend on the type of coordination and communication between the agents, as well as the type of agents that comprise the group. There are different approaches about organizational models, like [17] or [18].

From the perspective of this system, a VO-based MAS, PANGEA [19], has been used, which provides different tools to develop agents and deploy them in VO, and it is specially oriented to manage information obtained from the environment, including the information of multiple sensors. The characteristics of the agents systems allow this paradigm to be employed in a wide variety of research fields, such as data analysis in bioinformatics [20,21], classification of facial image classification according to gender and age [22], or WSA data fusion [18] but with a nexus that allows working with, and analyzing, large amounts of data. A large amount of data allows us to obtain useful information in very diverse areas and to recognize data trends (behavior patterns, consumption patterns, and spending patterns) [23]. In addition, the autonomy with which agents are provided allows them to interact with each other without any human intervention. Their ability to perceive changes in the environment and react to them makes multiagent systems an ideal approach for obtaining data from an environment and for responding to these changes with appropriate actions.
2.2. Rule-Based Reasoning Systems. A transformation between the observed parameters that are provided by multiple sources like sensors, and a decision or inference, produced by fusion estimation or inference processes, and the interpretation of the situations and the relationships among them are the most important characterization of information fusion. The interpretation of the data gathered from different sensors involves automated reasoning using explicit or implicit information based on knowledgebased methods such as rule-based reasoning systems [24]. The main reason to implement an automated reasoning system is to create a system that can make decisions based on new and previous information obtained by a network of heterogeneous sensors. There are different developed formal schemes based on fuzzy logic, formal logic, probabilistic reasoning, case-based reasoning, and other similar techniques. All of them define how to use the knowledge base to obtain conclusions or inferences, for example, with the use of rules, frames, etc. So, the main block for developing an automated reasoning system is the creation of a knowledge base and the rules associated with it. When it comes to data fusion, the situation refinement and threat detection are dominated by knowledge-based methods like the mentioned before [25]. In the developed system, information fusion algorithms are applied to combine the information gathered from each of the sensors through a case-based reasoning (CBR) mechanism [26]. CBR is a methodology, usually applied on knowledge-based systems, that learns from previous situations. CBR systems work with cases, which are the most important element of this kind of systems, and consist of a structure that stores problems, elements (cases), and its solutions. Therefore, each case can be interpreted as a database where different problems are stored with relationships with the solutions to every stored problem. This provides the system with the ability to generalize in order to solve new input problems. CBR systems have learning capabilities because of their own structure, which is composed of four main phases as defined by [27]: retrieval, reuse, revision, and retention.

2.3. Heterogeneous Sensor Networks. The use of sensor networks is growing rapidly because of the continuous technological advances. One of the most recent and extended concepts is Machine-to-Machine (M2M). The concept M2M is widely linked to the technological scope of Internet of Things (IoT) and Smart Cities, where not only people get connected; anything could be connected to the network. Smart objects are now occupying a wide part in our life. Any daily object can become part of a network without being limited to devices such as smartphones. This provides us with a general overview of the great volume of elements (sensors and actuators) that can connect, for example, all the elements behind a greenhouse. One of the interests of $\mathrm{M} 2 \mathrm{M}$ is to manage data in a stable and reliable manner, as well as unifying the different standards. This is the only way of making good use of the benefits offered by the technological evolution. With the huge variety of possible M2M communications, there are multiple possible configurations 


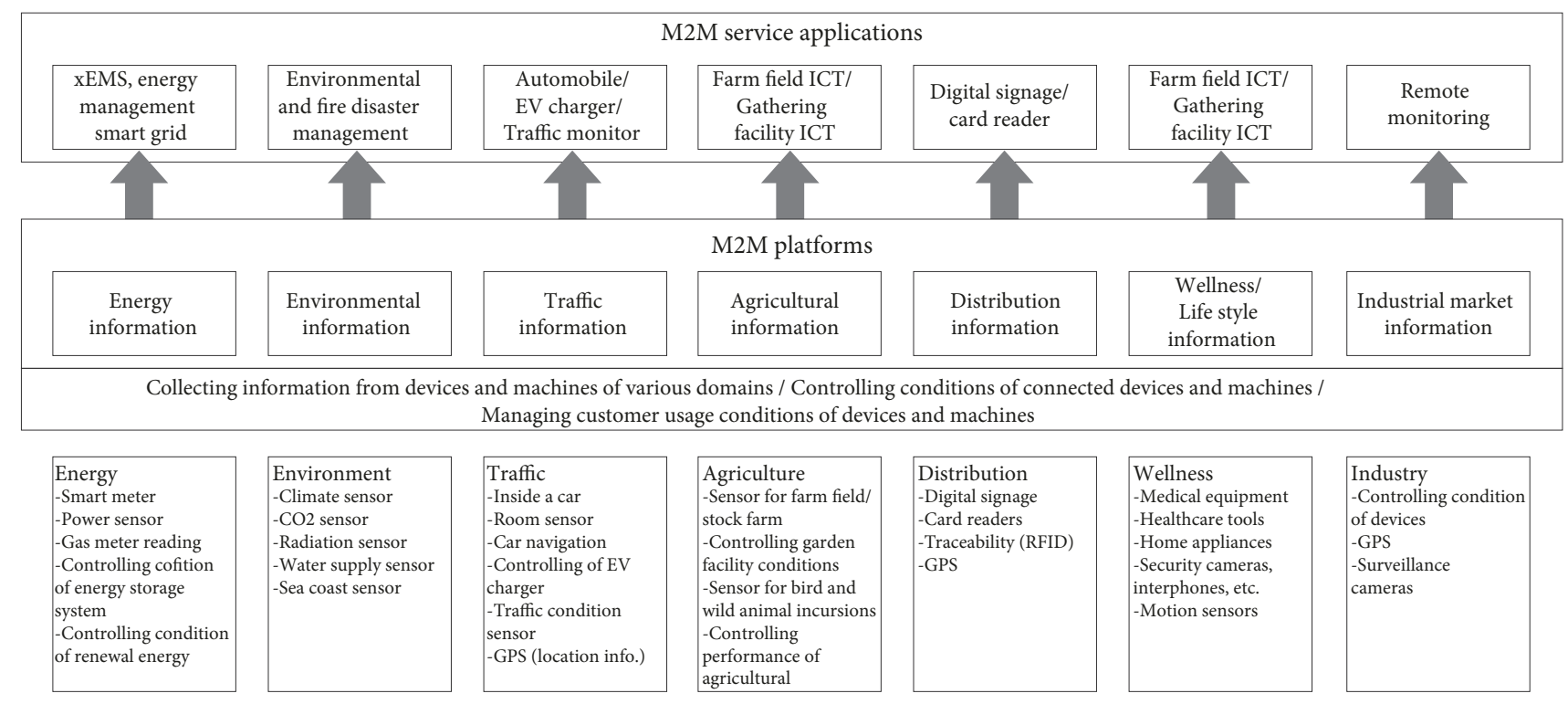

Figure 1: M2M applications.

regarding the technologies used to implement services, which can be combined to provide services. These services that M2M applications can provide are well reflected in [28] and they follow the diagram shown in Figure 1.

The main technologies that can be part of an M2M system are ZigBee, Bluetooth 4.0, Wi-Fi, Wi-Max, PLC, GSM/GPRS, 6LoWPAN, EnOcean, or Z-Wave [29].

2.4. Techniques and Infrastructure for Energy Recovery. To reuse the residual energy (hot water) of the power plants to heat the greenhouses, it is necessary to deploy a series of sensor systems that allow energy to be distributed efficiently. There is a model for using the energy wasted in power plants that allows the energy to heat the water in a tank and this water is also heated by means of a heat pump (powered by electric power of a solar farm). There are multiple approaches that have worked focusing on the use of ground heat exchangers (GHE) assisted by solar energy such as $[30,31]$.The flow of hot water generated by the hot exhaust gas that is extracted from the power plants is stored directly by a geothermal energy system that uses the internal heat of the earth to avoid energy losses while it is stored in the tank.

Hot water is used by the on-demand system to regulate the temperature of the associated greenhouses. When heated, the water is distributed through the greenhouses, more specifically, towards its Air Transfer Unit (ATU) where hot water is transformed into hot air to maintain the target temperature of each greenhouse. The water whose heat has already been collected is conducted by the ATU to a colder water tank, whose water is used as an input to recover energy from the hot exhaust gases wasted by the power plants.

\section{Proposed System}

To carry out the development of a system such as the one proposed, it is necessary to use an architecture that allows communication between all the elements that must make up the system. These elements are part of a distributed system in which each element must perform a separate process from the rest. This could be developed using a traditional object-oriented programming (OOP) methodology; however, decision-making would follow a number of previously established patterns. In order to make a realtime decision-making process evaluating the situation of the context in which the system is deployed, it is necessary to use an agent-oriented programming (AOP) methodology. The use of agents allows decision-making to be based on a BDI model (Beliefs, Desires, and Intentions) which means that the analysis of the context conditions, according to the agent in charge of the decision-making (energy use, energy distribution, etc.), is evaluated and adjusted to the conditions in real time in a more productive way.

The proposed multiagent system allows a management of the necessary infrastructure to implement the waste energy recovery system. The use of a system based on agents allows the different agents that make up the system to focus on the specific tasks, the communication with the infrastructure, the obtaining of similar cases (agents that implement a CBR system), or taking decisions (shipment of the amount of energy needed).

3.1. Infrastructure. The architecture reuses waste energy (hot water) from power plants to heat greenhouses; its subsystems and its connections are presented in this section.

Sensors required by the system are deployed in this infrastructure in order to distribute the energy in an efficient way, considering the greenhouse's requirements which are defined by the user by means of the software described in Section 4.3.

Figure 2 shows a block diagram of the infrastructure and the connections of its blocks. Two recycled energy inputs 


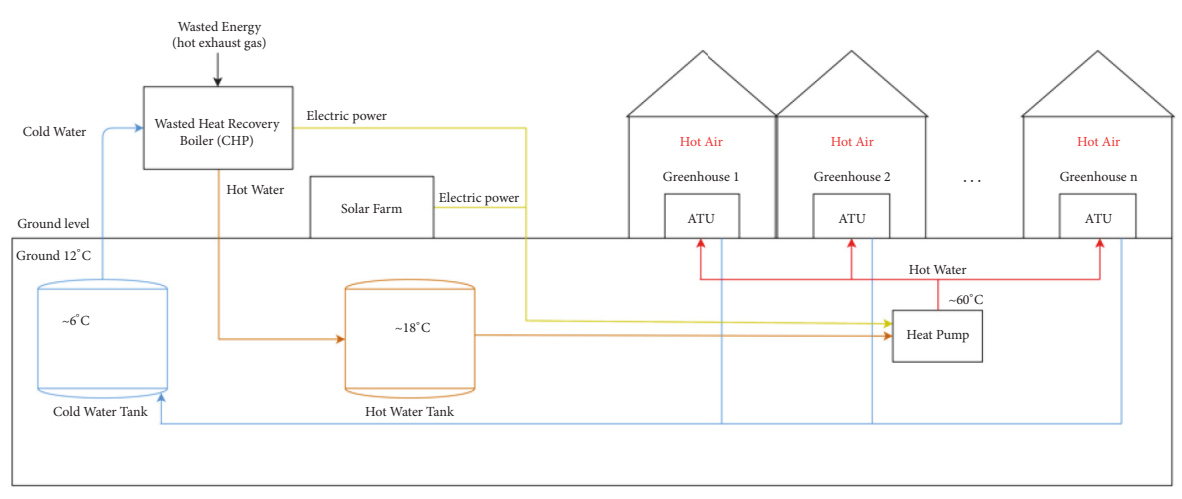

FIGURE 2: Energy recovery infrastructure schema.

are shown. On the one hand, the input coming from the waste energy generated by the power plant ("Waste Energy" in the image) is transformed into hot water and electric power thanks to a Combined Heat and Power (CHP) module. On the other hand, there is a solar farm to harness solar energy and increase the generated electric power that supplies other modules.

The hot water flow generated by the hot exhaust gas which is extracted from the power plants is directly stored by using a geothermal energy system that uses the internal earth heat to avoid energy losses while staying stored in the tank.

The standing water is used by the system on demand to regulate the temperature of the associated greenhouses. A heat pump is used to increase the water temperature from $18^{\circ} \mathrm{C}$ to $60^{\circ} \mathrm{C}$ approximately with the help of the power energy provided by the solar farm and the CHP. When heated, water is distributed across the greenhouses, more specifically, towards their Air Transfer Unit (ATU) where the hot water is transformed in hot air to keep the goal temperature of every greenhouse. Water whose heat has already been grabbed is drifted by the ATU towards a colder water tank, whose water is used as an input to recover the energy from hot exhaust gas wasted by the power plants.

3.2. Multiagent System. At the computing level, a MAS has been used to create the system. The use of this type of software architectures allows the development that can be efficiently adapted to the real scenario. This allows it to be implemented independently of variables such as size or number of greenhouses. In addition, MAS-based technology is designed specifically for environments in which information from the environment must be perceived in a heterogeneous manner, and artificial intelligence mechanisms are executed on this information to obtain a result adapted to the needs.

In addition, the use of used-vehicles allows large MAS to be structured internally in such a way that the functionality is decoupled, thus simplifying communication between the agents present in the system. In this sense, three used-vehicles have been included in the system described above, which are responsible for the functional part of the system.

For its development, PANGEA [19] has been used, which is a service oriented and VO-based platform that allows the implementation of open MAS to take maximum advantage of the distribution of resources. The main advantage is that it provides VOs for generic functionalities such as service discovery, standards definition, or data access. Therefore, the design and development of the system focus on the VOs of the functional part, which can be seen in Figure 3 and which are described below.

Workflow VO. This VO encapsulates the functionality related to the flow of information of the functional system. It runs two agents: (i) User Data Agent, which allows the necessary configuration to be inserted by the users who manage the greenhouses; (ii) Manage Workflow Agent, which allows the interconnection of the different functional parts, offering a series of services that they call agents of other organizations.

Greenhouse Data VO. This VOI encapsulates the functionality related to the needs and condition of each greenhouse. That is, for each greenhouse registered in the system, a new instance of used-vehicle is created, which contains the following agents: (i) Data Acquisition Agent, which is responsible for providing the mechanisms of access to the information that is handled, as well as making use of other services that provide information necessary for this used-vehicle; (ii) Consumption Agent, which implements the functionality related to the energy information of the system (including reused energy); (iii) Requirements Agent, which is in charge of managing the parameters required by the farmer and initiating the necessary actions to adjust them to your needs; (iv) Sensor Agent, which is an agent in each virtual organization for each sensor needed in that greenhouse; the number of sensors required is described in Section 4.3; (v) Actuator Agent, which is an agent in each virtual organization for each actuator required in that greenhouse. The number of sensors required is described in the Section 4.3.

Decision-Making VO. This VOI encapsulates the functionality related to the response that the software must perform on the environment to adapt to the needs at all times. This requires a number of decisions based on the data. The services of the agents present in this used-vehicle provide the result according to the parameters that depend on each greenhouse. The greenhouse information reaches the Decision-Making 


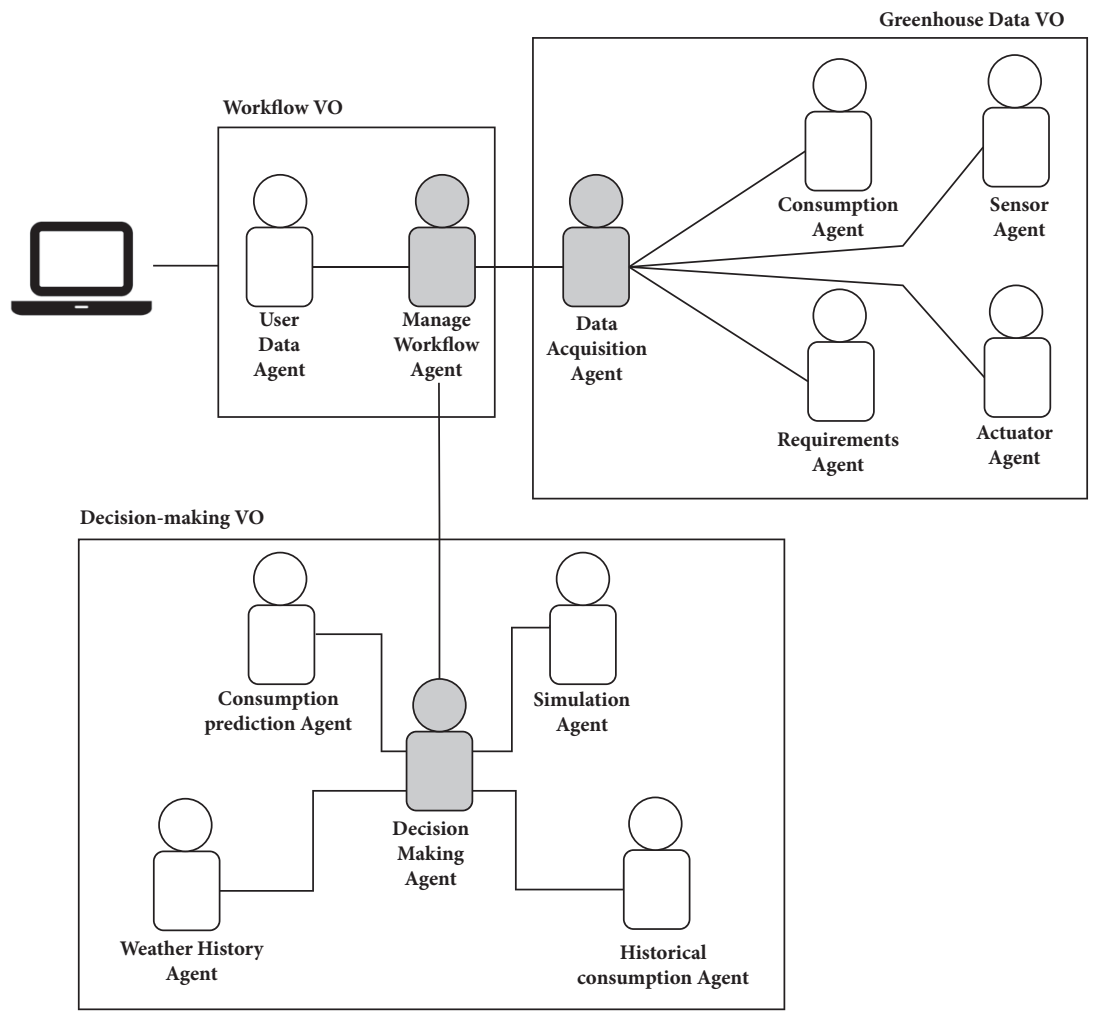

FIGURE 3: Architecture overview.

Agent, which is responsible for determining which agent(s) of your used-vehicle vehicle must be executed to achieve the objectives of the required action. Up to four types of actors may be involved in the decision: (i) Consumption Prediction Agent, which is responsible for making estimates of the consumption that will be needed to achieve the required parameters; (ii) Simulation Agent, which is responsible for providing a preview of the results to the farmer so that it can analyze its consumption over the long term; (iii) Historical Consumption Agent, which is responsible for managing historical information on cases, including a list of system predictions and actual values, in order to improve the accuracy of prediction systems; (iv) Weather History Agent, which is responsible for recording how past weather conditions have influenced predictions.

The following section describes the functioning of the agents related to the intelligent part of the system, which allows predicting the energy needed to meet the greenhouse's needs and thus is able to make an efficient distribution of the energy reused. More specifically, it explains the operation of the Historical Consumption Agent, which essentially implements a CBR system and the Consumption Prediction Agent, which uses a neural network to predict consumption based on similar cases recovered.

3.3. Estimation of the Energy Required by Each Greenhouse. When making predictions for an efficient distribution of recovered energy in the form of hot water, it is necessary to understand the functioning of the Historical Consumption Agent and the Consumption Prediction Agent. The former is responsible for implementing a CBR system based on other used-vehicle agents.

The CBR system, whose scheme is shown in Figure 4, starts its execution when in a greenhouse there is a lag between the parameters measured by the sensors and the specification of the needs made by the farmer through the software application.

Once the first stage of the CBR system is started, "Retrieve", the greenhouse data obtained from the corresponding Greenhouse Data VO (via the Workflow VO) are preprocessed. It processes them to detect the greenhouse architecture and retrieves from the case memory all previous cases of the corresponding structure. More specifically, there are three greenhouse structures used. In the figure, the $j$ structure types $i$ are recovered, with $j$ being one of the three types used and $j$ the number of previous cases in greenhouses with the same structure. The second stage, "Reuse", allows the quantity of energy required to satisfy demand to be estimated on the basis of the Consumption Prediction Agent. In the third stage, "Revise", the estimated energy is distributed in the previous stage and measured until the parameters required by the farmer are reached. Once you have the necessary real energy, which does not always have to be the same as that predicted in the second stage, you proceed to the fourth and last stage. At this stage, "Retain", the neural network of that type $\left(A N N_{i}\right)$ is retrained and the case memory is updated with the new case and the updated neural network $\left(C_{i . . j+1}\right)$. 


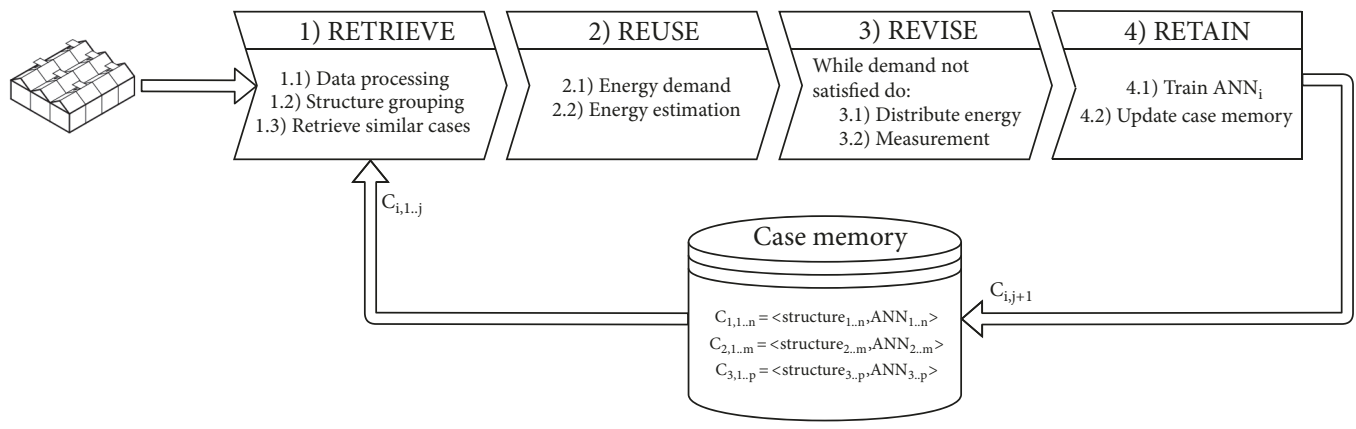

FIGURE 4: CBR cycle to predict the required energy.

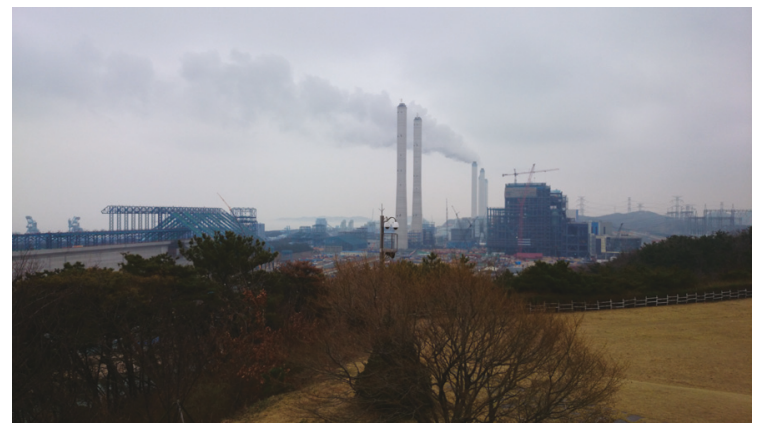

Figure 5: Infrastructure of the Dangjin power station.

Regarding the operation of the Consumption Prediction Agent, it is responsible for maintaining the neural networks used. Every cluster has a trained MLP (Multilayer Perceptron) where the inputs are target temperature, external temperature, internal temperature (initial), solar radiation, relative humidity, humidity ratio, ventilation, current weather station, and the architectural structure of the greenhouse itself, and the output is the amount of energy (kWh) required to move from the internal (initial) temperature to the target temperature. The selected activation function is sigmoidal and we do not want any extreme values for the training. In the middle layer $2 n+1$ neurons are placed where $n$ is the number of neurons in the input layer. This criterion is based on Kolmogorov's theorem [32].

The ANN is trained periodically both manually and automatically, by using a graphic user interface to facilitate the process. Automatic training is performed when a defined number of new cases is included in the system. In manual training, the evolution of the error is analyzed with a set of training data. When the error begins to reduce at a slower rate, the neural network is validated with the test data. As the training continues the error produced in the test data reduces.

3.4. Management User Interface. Final users can interact with the system thanks to the high level services provided by the software platform. In this way, there is a two-way communication among the involved parts to establish settings of all services of the system that can be used as well as services to get information on demand or in real time.
Therefore, in order to use those services properly, software applications are required in order to use them and interact with the final users. The software applications are adapted to the requirements of every case study and it can be designed as a web application, mobile application, SMS, or e-mail notification services, etc.

The main advantage is that programming heavy functionalities in every offered application is not required, as they only have to use the services that the platform provide. This significantly speeds up the processing load of the final applications; that could be as simple as an interface to cross data between the user and the platform.

\section{Case Study}

This section presents the components involved in the proposed system, the plant from which the wasted energy is reused, the greenhouses in which the energy is used, the infrastructure needed to recover the energy, and the MAS that manages all the components.

4.1. Power plant. The data will be used in terms of energy wasted by the Dangjin power plant, in Korea (Figure 5), in South Korea. The Dangjin complex comprises eight power plants that generate a total of 4,000 megawatts (MW), $500 \mathrm{MW}$ each of them. Specifically, the energy data wasted by one of the towers will be used.

4.2. Greenhouses. The energy wasted by the plant will be used in the reduction of the energy needs of four greenhouses with a cultivation area $\left(300 \mathrm{~m}^{2}\right)$ dedicated to the planting of peppers. All greenhouses have the technical characteristic systems: a rigid plastic (polycarbonate) double layer, two forced fans, seven wet pads, and a 30\% light reduction shade curtains, and a Booster Bov-500 heating $1500 \mathrm{~kW}$ also has heaters of $75 \mathrm{~kW}$ of power. Two of the four greenhouses have an architectural structure of type A-frame and the other two of type Arch. The cultivation of peppers requires very specific climatic conditions, this culture that can not be subjected to temperatures lower than $13^{\circ} \mathrm{C}$ and higher than $40^{\circ} \mathrm{C}$. The cultivation of peppers requires frequent irrigation but spaced in time (three or four days) just after being transplanted and every week of summer through drip irrigation, the humidity of the crop should not be excessive [33]. In Table 1 we can 


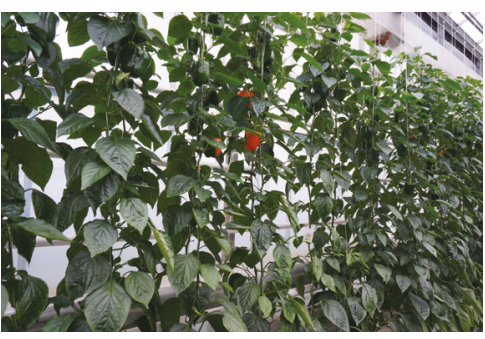

(a) Irrigation system in the pepper crop

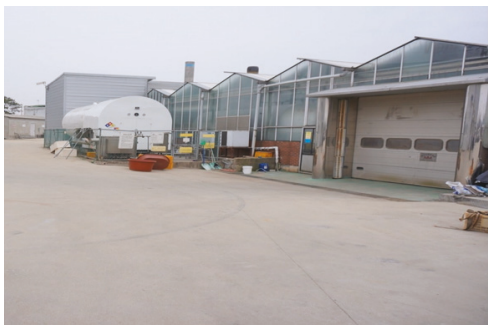

(c) Condensation water tank

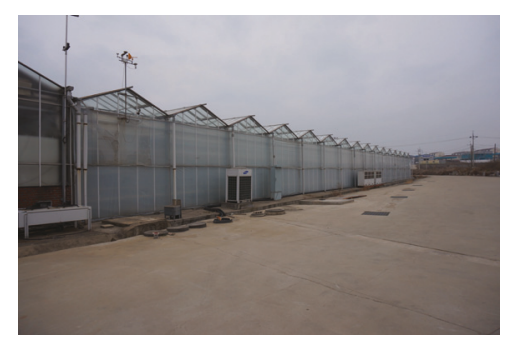

(b) A-frame greenhouse architecture

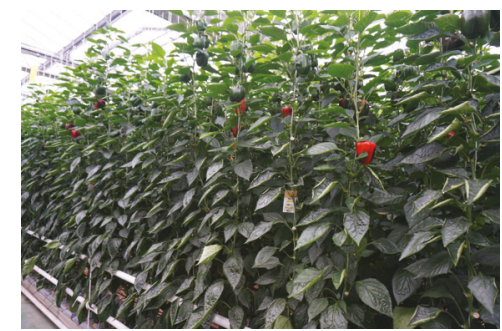

(d) Heater and evaporative cooling system

FIGURE 6: Case study greenhouse with pepper crop.

TABLE 1: Temperatures for the ideal growth of peppers.

\begin{tabular}{lccc}
\hline \multirow{2}{*}{ Cultivation stages } & \multicolumn{3}{c}{ Temperature $^{\circ} \mathbf{C}$} \\
& Optimum & Min & Max \\
\hline Germination & $20-25$ & 13 & 40 \\
Vegetative growth & $20-25$ (day) & 15 & 32 \\
& $16-18$ (night) & & \\
Flowering and fruit & $26-28$ (day) & 18 & 35 \\
& $18-20$ (night) & & \\
\hline
\end{tabular}

observe the characteristics in terms of temperature of pepper crop.

In Figure 6, we can observe the sixteen greenhouses of type A-frame with natural ventilation and force fans with a heater and evaporative cooling system. Within this group of greenhouses, the case study was carried out in eight greenhouses (four as a control group and four with the developed system).

4.3. Deployed Sensor and Actuator Network. Sensors deployed in the infrastructure are grouped in different types of networks. These networks use M2M techniques which communicate with each other and with the system. In this work, all the sensors are powered by the electrical network because of the advantages that it provides over the use of batteries when using static position sensors, so the communication energy efficiency is not a priority when designing the M2M algorithms. On the other side, data transmission uses wireless technology with the associated advantages when deploying this kind of communication networks.

In the developed system, BLE, Wi-Fi, and 6LoWPAN based networks have been used to connect all those sensors and actuators, allowing effectively controlling the system. 25+
$8 * n$ sensors have been used for the monitoring task (where $n$ is the number of greenhouses in the case study and 25 is the number of sensors that are needed to deploy the system). There are 6 different types of sensors as outlined in Table 2.

In addition to the sensors described previously, there is a number of valves which regulate parts of the system; one valve controls the gas input of the CHP and $8+n$ valves control the flow of water at the different stages of the system, where $\mathrm{n}$ is the number of greenhouses in the system. This ensures that the system is able to autonomously regulate the supply of water in order to establish the desired conditions.

4.4. Experimental Set-Up. The system has been evaluated for six months (September 2016 to January 2017); in this period in four greenhouses there has been a planting of peppers without using the system and in four other greenhouses through the waste energy recovery system. In this way all the greenhouses were under the same climatic conditions, autumn and winter in South Korea.

In the calculation of the air conditioning needs of each greenhouse $\left(Q_{c l i}\right)$ both the climatic conditions to which the greenhouse is exposed and the climatic conditions that it is necessary to keep inside for the correct development of the pepper culture are involved. The main climate data that characterize the climate and have been taken into account are maximum intensity of solar radiation, temperature and humidity outside, and wind direction and average wind speed. The main parameter in the energy balance of a greenhouse is the outside temperature, which directly determines the heating needs.

To calculate the energy needs needed to heat each greenhouse, data were taken from a winter day at 7:00 a.m. in the previous year, with no solar radiation and with a crop with two months of growth (evapotranspiration processes occur). The results of these measurements were Max. solar 
TABLE 2: Sensors used in the system.

\begin{tabular}{|c|c|c|c|}
\hline Sensor type & Function & Position & Number \\
\hline \multirow{7}{*}{ Thermal } & \multirow{7}{*}{$\begin{array}{l}\text { It measures the temperature of the water or the air in } \\
\text { the point of the system where it is placed. }\end{array}$} & $\begin{array}{l}\text { (i) } 2 \text { inputs (gas and water) and water output of the } \\
\text { CHP module. }\end{array}$ & 3 \\
\hline & & $\begin{array}{l}\text { (ii) Input and output (water) of the Hot Water Tank } \\
\text { module. }\end{array}$ & 2 \\
\hline & & $\begin{array}{l}\text { (iii) Input and output (water) of the Cold Water Tank } \\
\text { module. }\end{array}$ & 2 \\
\hline & & (iv) Input and output (water) of the Heat Pump module. & 2 \\
\hline & & (v) Input and output (water) of each ATU module. & $2 * n$ \\
\hline & & (vi) Air inside of every greenhouse. & $1 * n$ \\
\hline & & (vii) Outdoor temperature. & 1 \\
\hline \multirow{5}{*}{ Flowmeter } & \multirow{5}{*}{$\begin{array}{l}2 \text { different models are used: } \\
\text { (i) Measurement of the amount of gas flowing through } \\
\text { a point of the system. } \\
\text { (ii) Measurement of the amount of water flowing } \\
\text { through a point of the system. }\end{array}$} & (i) Gas input and water output of the CHP module. & 2 \\
\hline & & (ii) Output (water) of the Hot Water Tank module. & 1 \\
\hline & & (iii) Output (water) of the Cold Water Tank module. & 1 \\
\hline & & (iv) Output (water) of the Heat Pump module. & 1 \\
\hline & & (v) Input and output (water) of each ATU module. & $2 * n$ \\
\hline \multirow{5}{*}{ Manometer } & \multirow{5}{*}{$\begin{array}{l}2 \text { different models are used: } \\
\text { (i) Measurement of the pressure of gas in a point of the } \\
\text { system. } \\
\text { (ii) Measurement of the pressure of water in a point of } \\
\text { the system. }\end{array}$} & (i) Gas input and water output of the CHP module. & 2 \\
\hline & & (ii) Output (water) of the Hot Water Tank module. & 1 \\
\hline & & (iii) Output (water) of the Cold Water Tank module. & 1 \\
\hline & & (iv) Output (water) of the Heat Pump module. & 1 \\
\hline & & (v) Output (water) of each ATU module. & $1 * n$ \\
\hline \multirow{4}{*}{ Ammeter } & \multirow{4}{*}{$\begin{array}{l}\text { This kind of sensor measures the current in a point of } \\
\text { the electric part of the system. }\end{array}$} & (i) Output of the CHP module. & 1 \\
\hline & & (ii) Output of the Solar Farm module. & 1 \\
\hline & & (iii) Input of the Heat Pump module. & 1 \\
\hline & & (iv) Every ATU module & $1 * n$ \\
\hline \multirow{2}{*}{ Volumetric } & \multirow{2}{*}{$\begin{array}{l}\text { This kind of sensor measures the volume occupied by } \\
\text { the water of the tanks. }\end{array}$} & (i) Hot Water Tank module. & 1 \\
\hline & & (ii) Cold Water Tank module. & 1 \\
\hline Hygrometer & $\begin{array}{l}\text { This kind of sensor measures the humidity of a } \\
\text { greenhouse. }\end{array}$ & (i) Air inside of every greenhouse. & $1 * n$ \\
\hline
\end{tabular}

radiation intensity $542 \mathrm{~W} / \mathrm{m} 2,23.5^{\circ} \mathrm{C}$ Out. temperature, $64 \%$ Out. humidity, WE Wind direction, and $8 \mathrm{~km} / \mathrm{h}$ Avg. wind speed for all the greenhouses.

The terms that intervene in the energy balance of a greenhouse are indicated in the form of energy intensity. According to the First Principle of Thermodynamics, the energy gained by the system is balanced by the energy lost by it. Although each author considers a series of different parameters, we have defined it according to the following equation:

$$
S R+Q_{c l i}=Q_{c c}+Q_{r e n}+Q_{e v p}+Q_{s o i} l(W)
$$

where

\section{$S R$ is solar radiation}

$Q_{c l i}$ is heat energy to be supplied (Qcal) or removed

(Qref) from the greenhouse

$Q_{c c}$ is heat lost by conduction-convection

$Q_{r e n}$ is sensitive and latent heat lost by indoor air renewal

$Q_{\text {evp }}$ is latent heat consumed in evapotranspiration of plants and soil
$Q_{\text {soil }}$ is heat flow lost by conduction through the ground

The first part of the equation corresponds to the energy to be introduced into the greenhouse and the second part corresponds to the energy that is lost through the greenhouse air. The energy requirements of each greenhouse depend on the difference between the required indoor temperature (necessary temperature of the crop) and the outside temperature.

In order to calculate the amount of energy that is necessary to introduce in the greenhouses, the energy balance equation previously described has been used. This quantifies the amount needed to be used to have the temperature that allows the ideal growth of greenhouse peppers in the autumn and winter period.

Once the energy needs of each greenhouse are known, the agents that make up the MAS coordinate with each other to perform all the associated tasks, obtaining data from the sensors, executing the IF algorithm, executing the opening or closing of the valvula, and execution of a CBR agent cycle to obtain the amount of energy needed to meet the needs of each of the greenhouses. Next, the results of the experiment are presented for the case study. 
TABLE 3: Climate characteristics in a day of November in A-frame greenhouse of the case study.

\begin{tabular}{|c|c|c|c|c|c|c|c|c|}
\hline Time & $\begin{array}{c}\text { Out. Temp } \\
\left({ }^{\circ} \mathrm{C}\right)\end{array}$ & $\begin{array}{c}\text { In. Temp } \\
\left({ }^{\circ} \mathrm{C}\right)\end{array}$ & $\begin{array}{c}\text { Target Temp } \\
\left({ }^{\circ} \mathrm{C}\right)\end{array}$ & $\begin{array}{l}\text { Soil Temp } \\
\left({ }^{\circ} \mathrm{C}\right)\end{array}$ & $\begin{array}{l}\text { Out. SR } \\
\left(\mathrm{W} / \mathrm{m}^{2}\right)\end{array}$ & $\begin{array}{c}\text { In. SR } \\
\left(\mathrm{W} / \mathbf{m}^{2}\right)\end{array}$ & $\begin{array}{c}\text { Out. RH } \\
(\%)\end{array}$ & $\begin{array}{c}\text { In. } \text { RH } \\
(\%)\end{array}$ \\
\hline 1:00 & 4.9 & 5.7 & 24 & 9.6 & 2 & 1.3 & 74 & 84 \\
\hline 1:15 & 4.7 & 5.5 & 24 & 9.6 & 2 & 1.3 & 74 & 84.2 \\
\hline $1: 30$ & 4.3 & 5.1 & 24 & 9.5 & 2 & 1.3 & 74 & 84.5 \\
\hline $1: 45$ & 4.2 & 5 & 24 & 9.5 & 2 & 1.3 & 74 & 84.7 \\
\hline 2:00 & 3.9 & 4.7 & 24 & 9.5 & 2 & 1.3 & 74 & 85 \\
\hline 2:15 & 3.9 & 4.6 & 24 & 9.5 & 2 & 1.3 & 74 & 85 \\
\hline $2: 30$ & 3.7 & 4.5 & 24 & 9.4 & 2 & 1.3 & 78 & 87.8 \\
\hline 2:45 & 3.6 & 4.3 & 24 & 9.4 & 2 & 1.3 & 78 & 88.8 \\
\hline 3:00 & 3.4 & 4.2 & 24 & 9.4 & 2 & 1.3 & 78 & 89.2 \\
\hline
\end{tabular}

4.5. Experimental Results. The sowing of pepper is done traditionally in the months of January or February, transplanting the plant once the first leaves are born and the minimum temperature is $15^{\circ} \mathrm{C}$ (otherwise it will be necessary to cover the plant at night). The cultivation of the pepper requires a minimum temperature higher than $13^{\circ} \mathrm{C}$ at night and that is not maximum of $21^{\circ} \mathrm{C}$. Temperatures below $12^{\circ} \mathrm{C}$ at night result in slow growth and fallen leaves. As for temperatures, it has more critical needs than similar crops such as tomato or eggplant.

The cultivation of peppers is going to be done in the greenhouse, during the months between September and January. The winter temperatures in Korea are cold. So often the outside temperature is much lower than the ideal temperature of the crop, being necessary to introduce energy to heat the greenhouse.

The CBR agent performs a cycle, in which it uses the cluster associated with the architectural structure of each greenhouse (A-frame, Quonset, and Arch). The steps for greenhouse 1 are described below. Cases are obtained thanks to the Historical Consumption Agent that implements the CBR system, so the CBR obtains the cluster associated with the greenhouses of A-frame structure and through the input parameters: target temperature, external temperature, internal temperature (initial), solar radiation, relative humidity, humidity ratio, ventilation, current weather station, and the amount of energy needed normally to change the initial inside temperature to the target temperature (without the system). Table 3 outlines the input parameters collected by the deployed sensor infrastructure.

The temperatures in autumn in Dangjin are characterized by being low and in winter the minimum temperatures do not exceed $0^{\circ} \mathrm{C}$. With these conditions the pepper crops can not grow outside the greenhouse. The temperature at which the crop stops growing is $12^{\circ} \mathrm{C}$, so the difference between the outside temperature and the minimum temperature of growth is high. However, to achieve optimum growth of the peppers the ideal temperature should be $24^{\circ} \mathrm{C}$ during the day and although at night it can be somewhat lower, it can not be less than $13^{\circ} \mathrm{C}$ due to the lower solar radiation. In the eight greenhouses of the case study has been set that the target temperature being $24^{\circ} \mathrm{C}$ for the growth of the pepper culture is optimal, also allowing comparing the effectiveness of the system to have the same climatic characteristics inside and outside the greenhouse.

By introducing hot or cold air into the greenhouse, the temperature of the greenhouse increases or decreases, which means that less energy is needed to reach the optimum temperature of the crops. Thus, in the results of the three case studies, it shows the needs in terms of $\mathrm{kWh}$ that the greenhouses need before implementing our platform. In this way, the results are focused on showing the reduction in terms of kWh produced by the system.

To validate that the system developed provides replicable results in cases of similar studies that share similar characteristics, and when performing the simulation on a single case study, it is necessary to determine whether the experiment carried out is correct in terms of the methodology used. To this end, it is essential to apply statistical methods that determine their degree of certainty and meaning. To check the effectiveness of our system, we used Student's t-test and Levene's test, where $\mathrm{F}$ is the Levene Test for the equality of the variations and $t$ is the t-test for the equality of the means [34], as it is shown in Table 4. $p$ value (two tails) tells you if your correlation was significant at a chosen alpha level. The $p$ value is the probability of a given statistical model that when the null hypothesis is true, the statistical summary (as the difference of means between the sample of the greenhouses without the systems and of the greenhouses with the system) would be equal to or greater than the actual observed results. If its $p$ value is small, then the correlation is significant. These tests were carried out to evaluate the difference in means (electricity consumption in $\mathrm{kWh}$ ) and the variations between the data obtained before and after the implementation of the platform. In all cases, the $p$ value has been less than 0.05 , which shows that energy has been saved thanks to the platform based on a multiagent system.

\section{Conclusions}

This paper has presented an innovative approach through the use of a multiagent platform that allows us to reuse thermal energy wasted by the power plants; this allows us to optimize the economic cost in terms of energy consumption 
TABLE 4: Results of Student's t-test and Levene's test performed in the case study.

\begin{tabular}{lcccccccc}
\hline & \multicolumn{2}{c}{ Before platform } & \multicolumn{2}{c}{ After Platform } & & & & \\
& Mean & Std. & Mean & Std. & t & p value (2-tailed) & F & p value \\
\hline Greenhouse 1 & 3077.23 & 1105.43 & 854.30 & 542.90 & 17.124 & $\mathrm{p} \leq 0.001$ & 40.423 & $\mathrm{p} \leq 0.001$ \\
\hline Greenhouse 2 & 3066.11 & 1200.02 & 1067.05 & 999.61 & 12.143 & $\mathrm{p} \leq 0.001$ & 8.537 & $\mathrm{p}=0.004$ \\
\hline Greenhouse 3 & 2814.87 & 1086.98 & 808.62 & 466.05 & 16.093 & $\mathrm{p} \leq 0.001$ & 54.733 & $\mathrm{p} \leq 0.001$ \\
\hline Greenhouse 4 & 2785.09 & 1046.97 & 873.86 & 616.59 & 14.922 & $\mathrm{p} \leq 0.001$ & 42.147 & $\mathrm{p} \leq 0.001$ \\
\hline
\end{tabular}

of greenhouses by introducing this energy to reach the ideal temperature in the cultivation of peppers. The use of a multiagent system in this type of energy management problems provides us with a system to easily manage the conditions of the crop as well as manage the actions of opening and closing of valves in the infrastructure that manages the power generated by the plant. This work presents the hypothesis that there is a possibility of reusing the thermal energy wasted by a power plant, necessary to be able to manage a series of needs (capture of data from sensors, data analysis or decision-making, among others) that makes the development of an architecture based on agents necessary. The development of a multiagent system is based on the possibility of using self-organization and adaptation capabilities, allowing dynamic responses to unexpected situations of change. Another advantage that this approach gives us is the ability to detect potentially interesting situations, such as errors in the operation of the technology used in the infrastructure and to manage them as effectively as possible.

Greenhouse Data VO manages the needs of the crop and obtains data from the sensor network deployed in both the plant and the greenhouses used in the case study. According to the tests carried out, it has been of great help to use a multiagent system that makes it possible to take advantage of the energy wasted by one cent, so that it can be reused to increase the temperature of the inside of greenhouses, allowing energy and economic savings. The use of the system in addition to obtaining energy savings, since greenhouses stop consuming energy and therefore obtain an economic saving, allows reducing $\mathrm{CO}_{2}$ emissions. The use of Historical Consumption Agent, which implements a CBR system, has allowed obtaining the energy necessary to reach the set temperature for optimal growth of the crop (fixed at $24^{\circ} \mathrm{C}$ ), based on the parameters of solar radiation, humidity, outdoor temperature, indoor temperature, and soil temperature. As can be seen in the results obtained, the system has allowed the reuse of power plant energy, reducing the amount of energy required by the grid to obtain the ideal temperature of pepper cultivation. This process has been carried out while maintaining the ideal growing temperature of the crop at all times. As for the percentage of reduction in energy consumption in the present case study, a reduction of $30.68 \%$ in average savings has been obtained with respect to the four greenhouses in which the system was not used. We can see that the percentage obtained in the case study is a similar percentage to other study cases with similar characteristics, which have been developed with a similar methodology, as in [35]. In the previous work, three case studies were presented (depending on the meteorological station), which serve as a frame of reference for the correct evaluation of a virtual organizations approach to a multiagent system, and the application of these approaches in a culture.

Although the percentage of reduction in the grid's energy consumption is considerable, it is necessary to quantify the benefit that this produces in the electric energy bill. It is also necessary to study the economic cost of deploying the infrastructure. The study of the cost of deploying the infrastructure, together with the energy saving that it provides, allows us to know the time in which the cost of the proposed system is amortized.

\section{Data Availability}

The data used to support the findings of this study are available from the corresponding author upon request.

\section{Conflicts of Interest}

The authors declare that they have no conflicts of interest.

\section{Acknowledgments}

This research has been partially supported by the European Regional Development Fund (FEDER) within the framework of the Interreg program V-A Spain-Portugal 2014-2020 (PocTep) under the IOTEC project grant 0123_IOTEC_3_E and by the Spanish Ministry of Economy, Industry and Competitiveness and the European Social Fund under the ECOCASA project grant RTC-2016-5250-6. The research of Alfonso González-Briones has been cofinanced by the European Social Fund (Operational Programme 2014-2020 for Castilla y León, EDU/128/2015 BOCYL).

\section{References}

[1] I. Monedero, F. Biscarri, C. León, J. I. Guerrero, R. González, and L. Pérez-Lombard, "Decision system based on neural networks to optimize the energy efficiency of a petrochemical plant," Expert Systems with Applications, vol. 39, no. 10, pp. 9860-9867, 2012.

[2] E. Vine, N. Hall, K. M. Keating, M. Kushler, and R. Prahl, "Emerging issues in the evaluation of energy-efficiency programs: The US experience," Energy Efficiency, vol. 5, no. 1, pp. 5-17, 2012.

[3] J. J. Bonilla, J. M. Blanco, L. López, and J. M. Sala, “Technological recovery potential of waste heat in the industry of the Basque 
Country,' Applied Thermal Engineering, vol. 17, no. 3, pp. 283288, 1997.

[4] O. M. Al-Rabghi, M. Beirutty, M. Akyurt, Y. Najjar, and T. Alp, "Recovery and utilization of waste heat," Heat Recovery Systems and CHP, vol. 13, no. 5, pp. 463-470, 1993.

[5] B. Hu, H. Liu, R. Z. Wang, H. Li, Z. Zhang, and S. Wang, "A high-efficient centrifugal heat pump with industrial waste heat recovery for district heating," Applied Thermal Engineering, vol. 125, pp. 359-365, 2017.

[6] D. Wang, A. Bao, W. Kunc, and W. Liss, "Coal power plant flue gas waste heat and water recovery," Applied Energy, vol. 91, no. 1, pp. 341-348, 2012.

[7] E. Aarts and R. Wichert, "Ambient intelligence," in Technology Guide, pp. 244-249, Springer, 2009.

[8] M. Wooldridge and N. R. Jennings, "Agent theories, architectures, and languages: a survey," in Proceedings of the International Workshop on Agent Theories, Architectures, and Languages, pp. 1-139, Springer, 1994.

[9] N. B. Klinghoffer and M. J. Castaldi, Waste to Energy Conversion Technology, Elsevier, 2013.

[10] M. Esen and T. Yuksel, "Experimental evaluation of using various renewable energy sources for heating a greenhouse," Energy and Buildings, vol. 65, pp. 340-351, 2013.

[11] H. Tantau, Optimal Control for Plant Production in Greenhouses, Mathematical and Control Applications in Agriculture and Horticulture, 1991.

[12] M. D. Heidari, M. Omid, and A. Mohammadi, "Measuring productive efficiency of horticultural greenhouses in Iran: A data envelopment analysis approach," Expert Systems with Applications, vol. 39, no. 1, pp. 1040-1045, 2012.

[13] P. Bresciani, A. Perini, P. Giorgini, F. Giunchiglia, and J. Mylopoulos, "Tropos: An agent-oriented software development methodology," Autonomous Agents and Multi-Agent Systems, vol. 8, no. 3, pp. 203-236, 2004.

[14] C. Carrascosa, J. Bajo, V. Julian, J. M. Corchado, and V. Botti, "Hybrid multi-agent architecture as a real-time problemsolving model," Expert Systems with Applications, vol. 34, no. 1, pp. 2-17, 2008.

[15] M. N. Huhns and L. M. Stephens, Multiagent Systems and Societies of Agents, Multiagent Systems: A Modern Approach to Distributed Artificial Intelligence, vol. 1, 1999.

[16] N. R. Jennings and M. J. Wooldridge, "Applications of intelligent agents," in Agent Technology, pp. 3-28, Springer, 1998.

[17] O. Boissier and B. Gâteau, "Normative multi-agent organizations: Modeling, support and control, draft version," in Dagstuhl Seminar Proceedings, Schloss Dagstuhl-Leibniz-Zentrum für Informatik, 2007.

[18] S. Rodríguez, J. F. De Paz, G. Villarrubia, C. Zato, J. Bajo, and J. M. Corchado, "Multi-agent information fusion system to manage data from a WSN in a residential home," Information Fusion, vol. 23, pp. 43-57, 2015.

[19] C. Zato, G. Villarrubia, A. Sánchez et al., "Pangea-platform for automatic construction of organizations of intelligent agents," in Distributed Computing and Artificial Intelligence, pp. 229-239, Springer, 2012.

[20] A. González, J. Ramos, J. F. De Paz, and J. M. Corchado, "Obtaining relevant genes by analysis of expression arrays with a multi-agent system," in Proceedings of the 9th International Conference on Practical Applications of Computational Biology and Bioinformatics, vol. 375, pp. 137-146, Springer, 2015.
[21] J. A. Castellanos-Garz, J. Ramos, A. González-Briones, J. F. de Paz, J. A. Castellanos-Garzón, and A. González-Briones, "A clustering-based method for gene selection to classify tissue samples in lung cancer," in Proceedings of the 10th International Conference on Practical Applications of Computational Biology \& Bioinformatics, pp. 99-107, 2016.

[22] A. González-Briones, G. Villarrubia, J. F. De Paz, and J. M. Corchado, "A multi-agent system for the classification of gender and age from images," Computer Vision and Image Understanding, 2018.

[23] F. Doctor, H. Hagras, and V. Callaghan, "A Fuzzy embedded agent-based approach for realizing ambient intelligence in intelligent inhabited environments," IEEE Transactions on Systems, Man and Cybernetics, Part A: Systems and Humans, vol. 35, no. 1, pp. 55-65, 2005.

[24] D. John, Expert Systems: Design and Development, University of Akron, 1996.

[25] D. L. Hall and J. Llinas, "An introduction to multisensor data fusion," Proceedings of the IEEE, vol. 85, no. 1, pp. 6-23, 1997.

[26] I. Watson, "Case-based reasoning is a methodology not a technology," Knowledge-Based Systems, vol. 12, no. 5-6, pp. 303308, 1999.

[27] A. Aamodt and E. Plaza, "Case-based reasoning: foundational issues, methodological variations, and system approaches," $A I$ Communications, vol. 7, no. 1, pp. 39-59, 1994.

[28] O. Shigeru, "M2m and big data to realize the smart city," NEC Technical Journal, vol. 7, no. 2, 2012.

[29] H. Okkonen, O. Mazhelis, P. Ahokangas et al., "Internet-ofthings market, value networks, and business models: state of the art report," Computer Science and Information Systems Reports, Technical Report 39, 2013.

[30] H. Esen, M. Inalli, A. Sengur, and M. Esen, "Modelling a ground-coupled heat pump system using adaptive neuro-fuzzy inference systems," International Journal of Refrigeration, vol. 31, no. 1, pp. 65-74, 2008.

[31] H. Esen, M. Esen, and O. Ozsolak, "Modelling and experimental performance analysis of solar-assisted ground source heat pump system," Journal of Experimental \& Theoretical Artificial Intelligence, vol. 29, no. 1, pp. 1-17, 2017.

[32] L. Ming and P. M. Vitányi, "Kolmogorov complexity and its applications," Algorithms and Complexity, vol. 187, no. 1, 2014.

[33] A. González-Briones, J. Castellanos-Garzón, Y. Mezquita Martín, J. Prieto, and J. Corchado, "A framework for knowledge discovery from wireless sensor networks in rural environments: a crop irrigation systems case study," Wireless Communications and Mobile Computing, vol. 2018, Article ID 6089280, 14 pages, 2018.

[34] B. B. Schultz, "Levene's Test for Relative Variation," Systematic Zoology, vol. 34, no. 4, p. 449, 1985.

[35] A. González-Briones, P. Chamoso, H. Yoe, and J. M. Corchado, "GreenVMAS: Virtual organization based platform for heating greenhouses using waste energy from power plants," Sensors, vol. 18 , no. 3, 2018. 


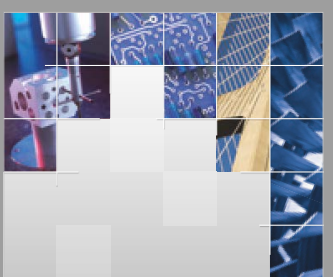

\section{Enfincering}
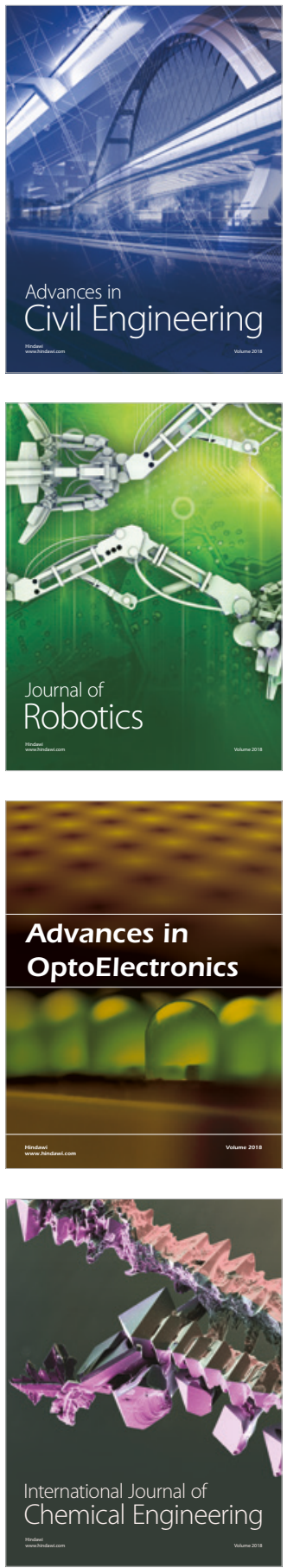

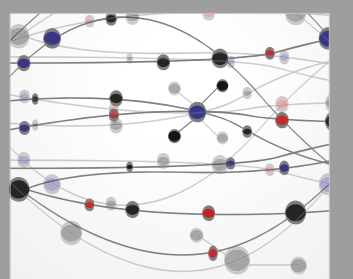

\section{Rotating \\ Machinery}

The Scientific World Journal

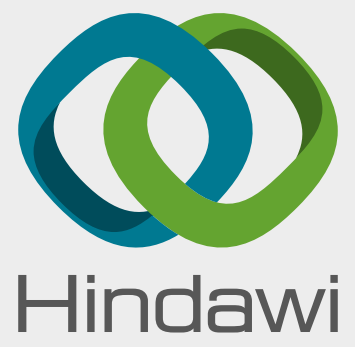

Submit your manuscripts at

www.hindawi.com
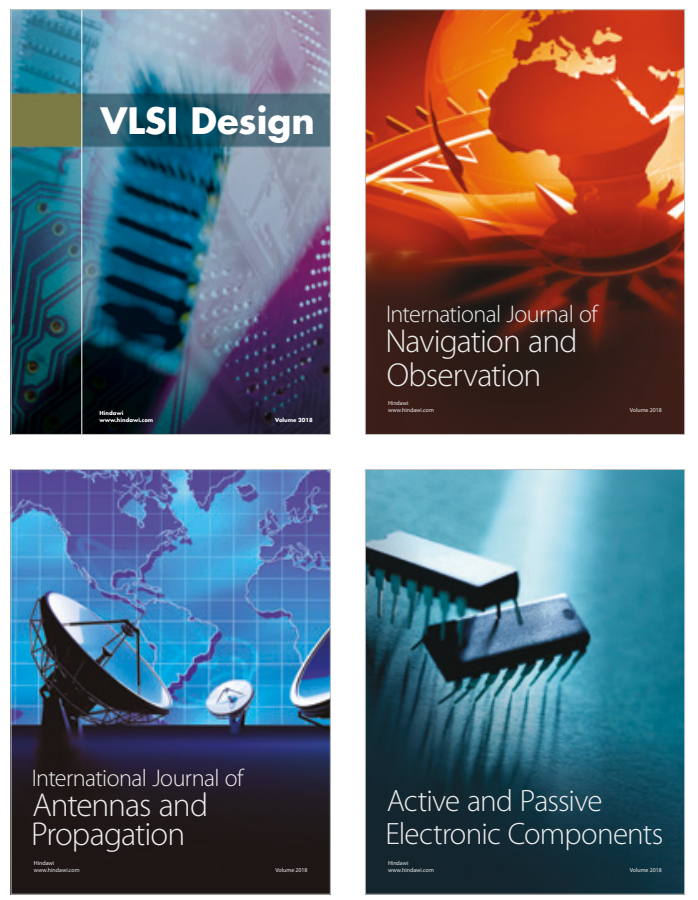
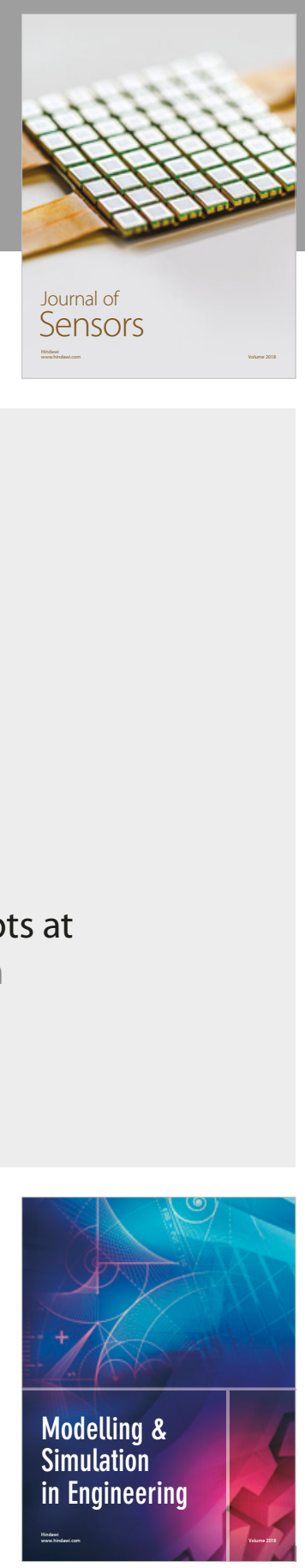

\section{Advances \\ Multimedia}
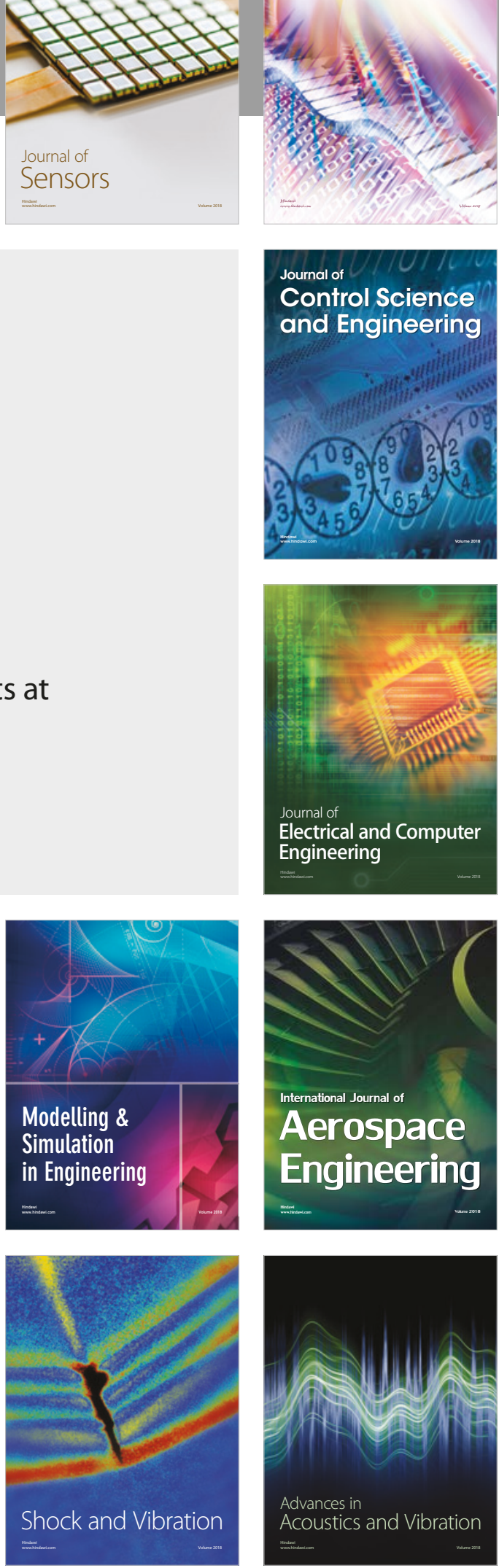